\title{
A decision-making support system for operational coordination of Home Health Care services
}

\author{
Liwen Zhang \\ DRI and Industrial Engineering Centre \\ Berger-Levrault and IMT Mines Albi \\ Labège, France and Albi, France \\ lliwen.zhang@berger-levrault.com
}

\author{
Christophe Bortolaso \\ $D R I$ \\ Berger-Levrault \\ Labège, France \\ christophe.bortolaso@berger- \\ levrault.com
}

\author{
Elyes Lamine \\ Industrial Engineering Centre \\ University of Toulouse-IMT Mines Albi \\ Albi, France \\ elyes.lamine@univ-jfc.fr \\ Mustapha Derras \\ DRI \\ Berger-Levrault \\ Labège, France \\ mustapha.derras@berger-levrault.com
}

\author{
Franck Fontanili \\ Industrial Engineering Centre \\ University of Toulouse-IMT Mines Albi \\ Albi, France \\ franck.fontanili@mines-albi.fr \\ Hervé Pingaud \\ CNRS LGC \\ University of Toulouse-INU \\ Champollion \\ Albi, France \\ herve.pingaud@univ-jfc.fr
}

\begin{abstract}
Nowadays, the majority of elderly people want to live longer in autonomy and well-being. As there are not enough places available in specialized institutions, Home Health Care Services are an alternative. With the fast rise of the demand, the organizations become aware of both existing limitations and of this potential for Research and Development (R\&D). They manifest two types of need: one is to be able to identify their own HHC coordination problem, the other is to find solutions for this specific need. In this paper, we propose a systemic analysis of these two aspects to identify the complexity of the relationship between the offer and the demand for HHC services. Then, we present one prototype BLPAD for decision-making support of the coordination in HHC. This research work reveals scientific obstacles and attempts to define a comprehensive response to this expectation of the HHC services ecosystem.
\end{abstract}

Keywords - Home Health Care, Coordination, Modelling, Operational Management, Decision Support, Systemic Analysis, BLPAD

\section{INTRODUCTION}

The WHO (World Health Organization) talks about purposes of human well aging. What does it mean? Elderly people who live longer should keep at least an intrinsic capacity of behaving and making their own decisions for a longer time, and have to do their best for the sake of being well concerned. More globally, the health system must adapt itself, because in a near future the worldwide demographic evolution will be driven by a significant increase in the number of people over 60 years old $(60 \%$ increase in the next 40 years), with a life expectancy that is expected to grow longer. Considering this context, the demands for delivering services to support independent elderly people who live alone at home are largely increased.

The challenge is to enhance networks of entities qualified to offer such support services. It is a priority in public policies for many countries. Organization for Home Health Care (HHC) is a support system profiled to face the challenge as it does not necessarily provide exclusively care, but also includes other categories of services like social, nutrition or recreative ones. In such a context, the care pathway concept is enlarged and becomes a health pathway or a life pathway, including a set of good care prevention and well-being practices. However, facing the large population that will call for such supports in a short time, as well as the diversity of demands to be satisfied, HHC organizations have to anticipate the phenomenon and be prepared to shift towards a more adapted capacity of actions.

If the HHC structures spend time and energy to respond at their best to the current market needs, they are directly and very concretely facing difficulties in terms of business process design and control for the future. This could drastically limit their quality of service over time if anything is done. But the inherent complexity HHC services have to face is an explanation of the large amount of research work related to the problem.

For example, the integration of liberal health practitioners who have contracted with such networked structures is a key point in system analysis. These health care providers, because of heavy daily workloads and a natural degree of freedom to decide what has priority and what has less priority at any time, are sources of uncertainty for the planning of HHC structures into which they have to operate. Very frequently, HHC are non-hierarchical organization types asking for a coordination capacity to be managed. The operational coordination we are going to define has a great importance in controlling such a human based support system. Moreover, as daily home-based services deliveries are subject to geographical features, the area where HHC is performing operations is generally limited in space. The operational coordination is therefore helpful to master the multiple transportation activities linked to service requests in a system that is distributed.

In this respect, our work is an attempt to clarify the concept of operational coordination of home health care through a systemic approach. Our approach begins with a literature review of the available knowledge about this subject. Then, on this basis, the definition of a framework, into which engineering tools will help to make HHC operational coordination becoming a reality is studied. It will be called the $\mathrm{HHC}$ coordination system in the following.

In this context, we first try to analyze previous works about coordination. Using a system engineering perspective, some unsolved problems will be identified that must be solved to address correctly the design of computer aided coordination tools. Finally, a prototype called BLPAD (French abbreviation: Berger-Levrault: Prise en charge A Domicile) is introduced. We will explain the software architecture of BLPAD and try to show why it could be progress for HHC management with respect to the level of future requests for services.

\section{CAPITAl KNOWLEDGE ON COORDinAtion OF HHC}

In the theory of coordination Malone and Crowston advocate that « coordination is about managing dependencies between activities for an explicit purpose ». In this sense, a business process design helps to better understand the concept. This vision has been extended to the case of coordination purpose for collaborative systems, using also a business 


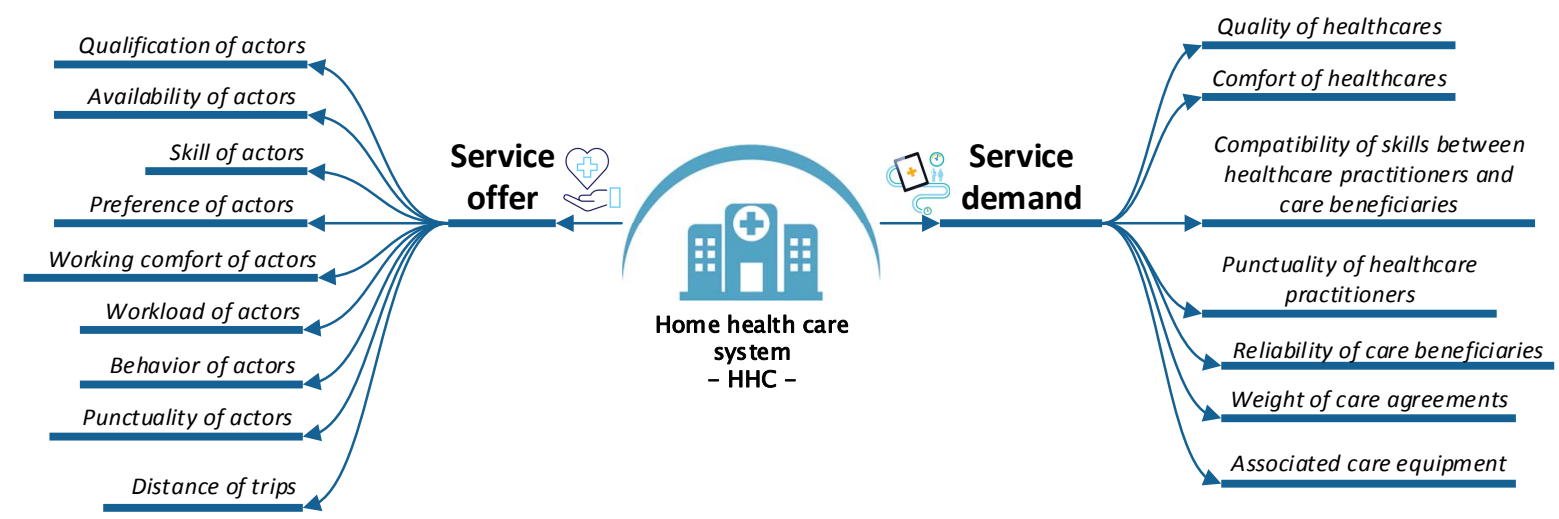

Fig. 1. Service offer and demand for HHC system with mind mapping presentation

process based frame by Rajsiri et al. [1]. Considering management of supply chains, coordination was early defined by Hill and Scudder sharing the same view(i.e. activities leading to an outcome) [2]. These authors said: " companies working jointly with their customers and suppliers to integrate activities along the supply chain to effectively supply product to customers». With the same purpose of offering the best service to users, McDonald et al. [3] provided a definition of coordination in the healthcare domain taking care about the diversity of actors involved: " coordination is the deliberate organization of health care activities to a patient by various actors in the health system. It involves the availability of staff and other resources. It is often performed by the exchange of information between care stakeholders ».

The coordination is therefore an activity of decision making in the context of a collective non-hierarchical structure with a twofold issue. One objective is to plan a set of activities to deliver local services needed by each person included into the HHC portfolio in conformity with the specification of the services the person must receive. Another objective is to set up the plan for each caregiver with respect to the constraints of his/her agenda. As classically expected in operations management, Gourgand [4] introduced three classic levels of decision-making (strategic, tactical and operational) with different time horizons to address planning and scheduling problems. We will only focus on the operational decision level when the healthcare practitioner team is known, and the service consumer portfolio is also given. Such a set of decisions are made on a short-term horizon going from one day to one week.

In order to go deeper into the knowledge of an HHC coordination system, we adopt three dimensions of a systembased analysis as is commonly practiced for separation of concerns when the subject is indeed complex. We propose to investigate the following dimensions:

- System modeling: The goal is to set the stage at a generic level using business objects implied in an HHC coordination system. We try to deal with the complexity of coordination issues using business process models. Among the design entities involved in this modeling exercise, we will distinguish many classes like, for illustration, the characteristics of the structure, the staff engaged in the realization of tasks (administrators, caregivers, and other stakeholders), the patients, the means of transport, etc.

- Specification of the short-term management: HHC coordination problems have been addressed by many different mathematical formulations. The relevant decision-making problems search for the value of many variables that will represent formally what to do, when to do it and how to perform it. In fact, many authors have studied sub problems of coordination separately, such as the scheduling of the care services, the routing of the caregivers, or the assignment of the caregivers to the HHC's requests of the patients on time scales that cover different periods (day, week, month). Such works show explicitly many facets of what has to be more or less formally decided by whom is willing to coordinate. We will conceive a decision frame to capture all these configurations of problems from objective functions to constraints to be satisfied.

- Architecture of a decision support solution: In this part, we focus on a decision support subsystem embedded in an HHC coordination system. In the literature, optimization methods are often applied to solve the formulated problems. Globally, the valuable judgment of an organization in charge of the control goes through a criticism about the quality of its decisions considering an estimation of the expected properties of the system in operation. The consideration of random variation, for example, is an important factor in the $\mathrm{HHC}$ due to the nature of some of the more lavish care activities.

\section{A. System modelling}

There is a large body of knowledge available on HHC coordination system. We highlight here a compilation of past contributions of literature and try to describe the broad range of variables and dependencies to be included in a system model.

According to En-nahli et al.[5], modeling the HHC coordination problem is complicated by many factors such as variation in the care beneficiaries' demands, the qualifications of the healthcare practitioner, the compatibility or affinity of skills between the care beneficiaries and the caregivers, unpredictable resource unavailability, daily rest times for healthcare practitioners, restrictions on healthcare caregivers' workloads, organizational requirements and fairness of the caregivers' workloads.

Fig. 1 is an entry point to our approach, a mind mapping based on the relationship between offer and demand for HHC. This classification gives a first distribution of the modeling elements in two parts, one featuring the actors delivering the services, the other qualifying the expectations of the beneficiaries. In each part, a branch explicates data on an HHC 
organization, decision variables for $\mathrm{HHC}$ coordination, as well as criteria for judging the validity of the services offered or even advanced relationships between the data and the variables that will be involved in the mathematical formulation of constraints to be satisfied.

The level of consideration about knowledge of an HHC system will be a determinant to explain the dynamics of the system in operation in a given context. The scientific literature clearly shows that intensive knowledge is needed and often proposes classifications for this purpose.

Castillo-Salazar et al. [6] highlight the affectation relationship of caregivers to final user demands. HHC coordination decisions are classified into four levels according to different time horizons classically known in hierarchical production management approaches, from strategic to operational [9][10]. Here, the operational level is split in two levels: a design level and an adaptation level. According to a study of Mankowska et al. [9], the weight of caregiver qualifications has to be considered for selecting the services to be made in HHC. Lanzarone et al. [10] elicits the criteria for classifying patients. Carello and Lanzarone [11] introduce the classification of caregivers according to the needs of patients.

The culture the regulation laws and the care practices of a given territory influence the HHC coordination system. Yuan and Fügenschuh [12] present the specificities of services offered by a HHC structure in Germany.

A nomenclature of procedures for caregiving is prescribed by regulation laws. It is an essential source for the exact definition of care activities. If this nomenclature is useful in the context of health insurance to guide the control of financial aspects, it provides a solid basis to build relationships between care procedures and caregivers' qualifications.

As an entry point, the relationship between offer and demand is natural and the critical nature for people of care services must be considered at the proper level. The human factor is, in some sense, minimized in past works. However, the medical world today is reticent to give greater weight to this action in planning the care activities and making the operational coordination more efficient. Thus, giving weight to this dimension could decrease the autonomy that the caregivers need to spend the necessary time with each patient. This is a point that must be taken into account in a desire to be quite exhaustive in the development of modelling tools for these systems, and therefore in any language adapted to the description of an HHC system for decision making.

\section{B. Specification of the short-term management}

Under the assumption that we would know how to characterize an HHC, whatever it may be, we must now address the implication of the management in this organization and the necessity of HHC coordination between stakeholders. In the same logic as in the previous section, we enumerate the knowledge related to the specification of the short-term management collected in the scientific literature.

Mankowska et al. [9] deal with the coordination through a daily planning model of care services performed by HHC staffs. En-nahli et al. [5] present a study aimed at finding feasible work schedules for each resource based on a daily horizon in order to ensure patients' and caregivers' satisfaction by controlling costs and respecting patients' preferences. These articles show the specific constraints in the daily planning of care services, the granularity in the definition of patients' needs, the individual qualifications of staff, the interdependencies between the different operational services and the grouping/ungrouping of caregivers.

According to Trautsamwieser and Hirsch [16], the main decision is the assignment of each care service to a caregiver during the week. For Yuan and Fügenschuh [12], the planning objective is to cover all weekly visits with minimal operational cost. This must be done without loss of service quality, with the guarantee of a sufficient duration of intervention, respect of the specified day and time intervals for a patient (availability, etc.). Rest and Hirsch [17] and Shao et al. [18] present an optimization problem by a case study, which is based on the coexistence of a daily model and a weekly model. The assignment constraints, including maximum working hours and break times, must be respected. These articles address the coordination in terms of the assignment of resources (weekly nurses' visits, caregivers' constraints, etc.)

Hiermann et al. [13] present a scheduling of caregivers based on constraints: the preferential schedules, the satisfaction of staff and the itineraries in a multimodal environment (cars or public transport) with an important Austrian HHC structure.

Redjem and Marcon [19] present a solution for planning the routing of caregivers in order to offer the best service and without violating patients' calendar preferences. Du et al. [15] identify the caregiver routing problem as a variant of the travelling salesman problem (TSP) [20].

Several insights have been learned by analyzing these sources. First, there are several relatively classic forms of problems that intertwine in this specification about the decision making needed to coordinate an HHC. Inspired by the vocabulary of production management, four types of decisions can be identified: planning, assignment, scheduling and routing. Second, it is appropriate to discuss the perspectives related to the decision. It seems logical that a total satisfaction of patients' expectations should be opposed to an ability to provide the expected service mainly due to resource limitations. This goes to a form of balance between offer and demand that any model must represent. Finally, the time horizons do play a key role. The day and the week are often the preferred time intervals, probably because of the limitations in judging the care provided to a patient in the medium and long terms.

\section{Architecture of a decision support solution}

The art of operational research is applied to these coordination problems identified previously. In view of the structuration of problems in this specialty, the coordination of the HHC has obvious similarities with the production management system (i.e. activity planning), with the skills management of human resources (i.e. skills assignment to activities) and also with the routing problems (i.e. TSP).

Given the mathematical variables characterizing the decisions, the preference attributed to one or more criteria, offers and demands constraints that are allowed, a range of solutions can be found. The algorithm and the resolution mode chosen are often very suitable for a coordination problem. Therefore, it is obvious that the size of the problems in the field leads to the difficulties of computation time and relativizes the operational scope of many attractive solutions in theory.

An elegant possibility to overcome this difficulty is to decompose the HHC coordination problem in several subproblems solved sequentially. The output of a formulation will provide bounds or new constraints for the next one. This decomposition approach has a price, which is the price of a potential sub-optimal solution when it is found. The advantage is the possibility to solve the problems with a larger size, which partly compensates for this observation. Table 1 illustrates the profusion of solutions through the literature, and also proposes a qualification of these studies using six criteria 
TABLE I SYNTHESIS OF DECISION SUPPORT FOR THE COORDINATION

\begin{tabular}{|c|c|c|c|c|c|c|}
\hline Reference & 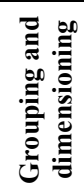 & 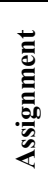 & 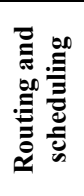 & 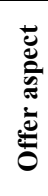 & 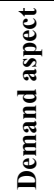 & Algorithmic basis \\
\hline Trautsamwieser and Hirsch [16] & $\mathbf{X}$ & $\mathbf{X}$ & & $\mathbf{X}$ & & Branche-Price-Cut \\
\hline Issabakhsh et al. [21] & & & $\mathbf{X}$ & $\mathbf{X}$ & & Robust solution \\
\hline Carello and Lanzarone [11] & & $\mathbf{X}$ & & & & Cardinality-constrained assignment model \\
\hline Du et al.[15] & & & $\mathbf{X}$ & $\mathbf{X}$ & & Genetic algorithm and local research \\
\hline Shao et al.[18] & $\mathbf{X}$ & & $\mathbf{X}$ & $\mathbf{X}$ & $\mathbf{X}$ & Mixed-variable programming \\
\hline Yuan et al.[22] & & $\mathbf{X}$ & & $\mathbf{X}$ & & Branch and bound, etiquette algorithm \\
\hline Lanzarone et al.[10] & & $\mathbf{X}$ & & $\mathbf{X}$ & & Markov Chain \\
\hline Mankowska et al.[9] & & & $\mathbf{X}$ & $\mathbf{X}$ & & Heuristic \\
\hline En-nahli et al.[5] & & $\mathbf{X}$ & & $\mathbf{X}$ & & Linear programming in mixed variables \\
\hline Rest and Hirsch [17] & & & $\mathbf{X}$ & $\mathbf{X}$ & & Tabu research \\
\hline Yuan and Fügenschuh[12] & & & $\mathbf{X}$ & $\mathbf{X}$ & & Multi-commodity, greedy construction and local search \\
\hline Redjem and Marcon[19] & & & $\mathbf{X}$ & $\mathbf{X}$ & & Greedy Heuristics \\
\hline Hiermann et al.[13] & & & $\mathbf{X}$ & $\mathbf{X}$ & & $\begin{array}{l}\text { Constraint programming, random procedure, memetic algorithm, } \\
\text { dispersion search and simulated annealing }\end{array}$ \\
\hline Rodriguez et al.[23] & $\mathbf{X}$ & & $\mathbf{X}$ & $\mathbf{X}$ & & Integer linear stochastic programming \\
\hline Yalçındağ et al.[24] & & $\mathbf{X}$ & $\mathbf{X}$ & $\mathbf{X}$ & & Kernel regression, k-nearest neighbor search \\
\hline Maya Duque et al.[14] & & & $\mathbf{X}$ & $\mathbf{X}$ & & Multi-objective approach \\
\hline
\end{tabular}

by inheriting the structuring dimensions of the coordination problem mentioned in the modelling part and in the specification part.

\section{BLPAD: PROTOTYPE FOR DECISION-MAKING SUPPORT OF THE OPERATIONAL COORDINATION IN HHC}

It seems that an exhaustive consideration of the multiple dimensions of the HHC coordination problem still remains an open subject of research. A proof of concept named BLPAD is described here. BLPAD helps to generate the daily planning of services, which consist of both the routing and scheduling results to satisfy a known demand in a given HHC structure.

BLPAD addresses the problem through the interaction of two HHC coordination modules: DefPAD, GenPAD. The module DefPAD is used to characterize the knowledge required from the HHC system in order to define what is $\mathrm{HHC}$ coordination, while the module GenPAD is used to generate an optimized coordination solution (scheduling and routing solution for all types of actors) for the given planning horizon. Fig. 2 shows the metamodel for the conception of the prototype BLPAD. However, we don't consider the break during each tour of the caregivers or the synchronization between two caregivers for executing one care service. Furthermore, the location of all the stakeholder and the HHC structure is characterized by the GPS coordinates.

\section{A. DefPAD: Modeling tool for HHC system}

DefPAD is based on a knowledge model of an HHC system. It has a graphical interface by which a user can easily provide information. This module is used to 1) create an HHC structure with its property, 2) define the care acts taxonomies and distribute caregiver roles, 3) be in charge to manage caregivers, such as their business abilities and special requirements, 4) be in charge of patient management, such as their personal healthcare services information and special needs, and 5) be in charge of care management, such as the care service indicator and the specification of the care plan. In conclusion, the main concepts concerning the offer side defined in DefPAD are HHC structure and caregivers. For the demand side, they are patients and associated care plans.

To characterize the HHC structure, we need its geographical address and all the care acts proposed by the structure, with the following information: the denomination of the care acts (i.e. changing a dressing), the time required to perform the care acts and the qualification required to perform the care acts (i.e. nurse).

To characterize the caregivers hired by the HHC structure, we need their first and last names, their qualifications (i.e.

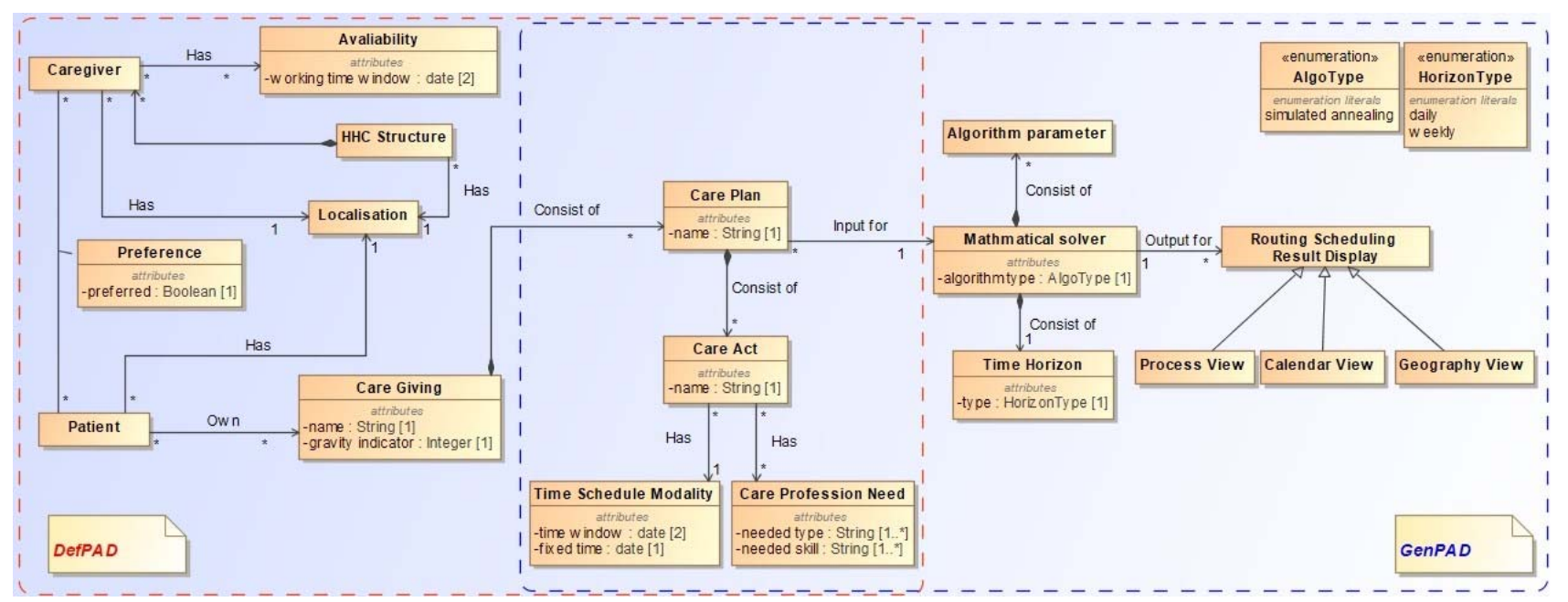

Fig. 2. Metamodel for the conception of the prototrype BLPAD 


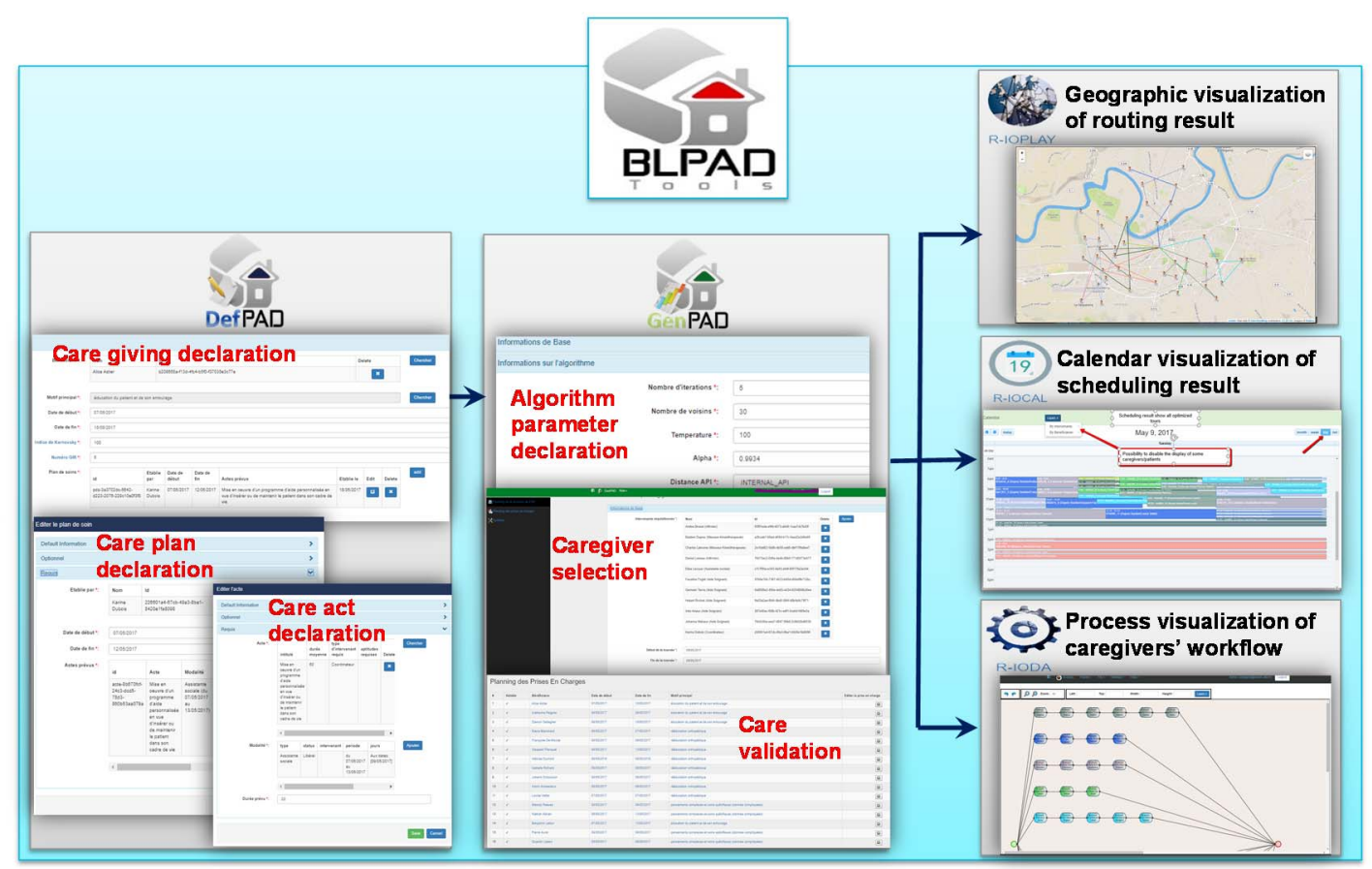

Fig. 3. Fonctional architecture of BLPAD and screenshoot of each module

assistant nurse), the schedules at which they work, which can be personalized (i.e. $8 \mathrm{am}-3 \mathrm{pm}$ and/or $3 \mathrm{pm}-7: 30 \mathrm{pm}$ ).

To characterize the patients registered in the HHC structure, we need their first and last name and their address.

The relationship between the caregivers and the patients we define as a concept named "preference" to guarantee the good coordination before reaching the planning stage, especially the uncertain events between the stakeholders. For example, a patient needs the special caregiver to execute his her requested care giving, or a caregiver has the allergic issue that makes her/him unable to perform some requested caregiving.

Finally, to characterize the care plans applied to the registered patients, we need the care act to be performed on given dates and its execution duration accurately, the schedule that they want to receive the requested care acts, such as the time windows and the fixed treating time. Furthermore, various medical indicators to measure the gravity of each care act are required. The set of care plans belong to the care giving requested by the patient.

Some information entered into DefPAD only concerns a specific HHC structure for one working day and shall be adapted when the day changes.

\section{B. GenPAD: Solution generator for HHC organization}

GenPAD runs with the objective of the optimum scheduling and routing results generation and the results visualization through different views, in order to provide the decision-making support to the HHC coordinator such as the start time of each care service and the sequence of the tour for each working caregiver. On the functionality side, GenPAD is aimed at: 1) generating the potential care services to be performed, based on the care plans declared within the DefPad module, 2) validating the care of each patient, 3) selecting the caregivers who are ready to perform the care services, 4) optimizing the daily planning based on the mathematical solver, and 5) visualizing the results under three different views.

GenPAD firstly queries the database which has been filled by DefPad to retrieve all the data on care services necessary for $\mathrm{HHC}$ coordination purpose: HHC structure information, a list of all the care plans to be performed and a list of all the available caregivers in the structure. Then GenPAD suggests the user select a time horizon he/she would like to plan (daily or weekly). At this stage, the coordinator can also choose to disable some of the caregivers they do not wish to mobilize. Afterwards, the mathematical solver can be executed with all these required parameters. GenPAD saves the most optimal

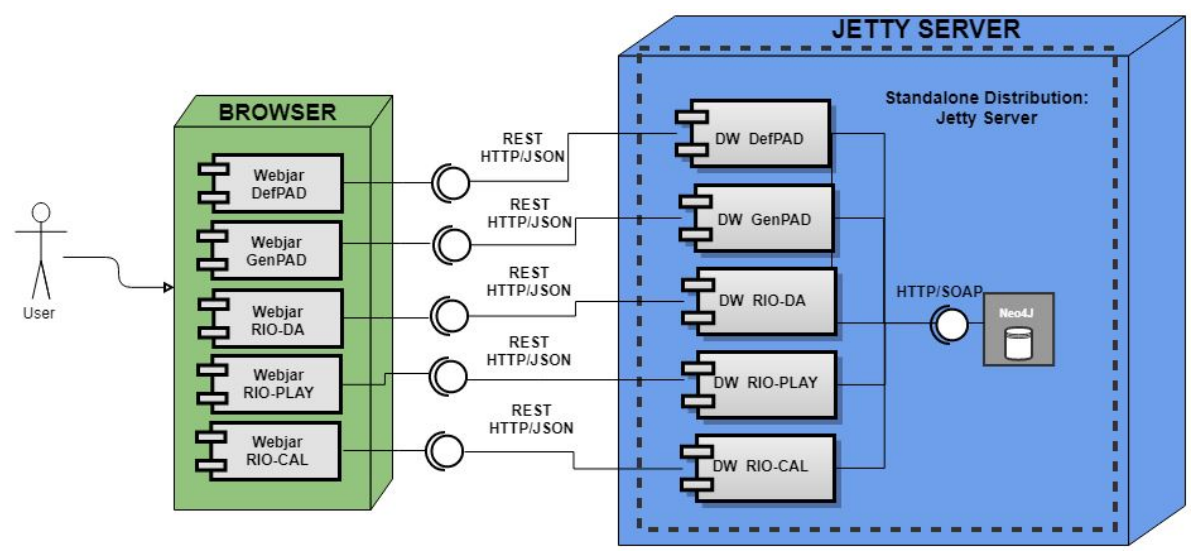

Fig. 4. Software architecture of the prototype BLPAD 
solution at the end of the process. The result will be displayed by the three modules, which allow the coordinators to read and manipulate the routing and scheduling results in three different views as their preferences: 1) geographical view for visualizing the routing result.Tthe objective is to display the order to execute each care services and the geographical distribution of the location for all the care services to be executed, 2) calendar view for visualizing the scheduling result, especially the start time, end time and the assigned caregivers for each of the care services, and 3) process view for visualizing the caregivers' workflow during his tour of the working day. Fig. 3 show the functional architecture of BLPAD and the screenshot of each module.

For the mathematical solver, we adopt the simulated annealing metaheuristic to achieve the routing and scheduling optimization based on a metaheuristic oriented mathematical formulation, taking into account the total satisfaction of all the stakeholders in the HHC structure. The formulation is presented in our recent research work [27].

Fig. 4 shows the software architecture of the prototype BLPAD. We adopt AngularJS to develop the user interface and our data structure is formatted as JSON to record the data entered through the interface. Furthermore, the REST standard is used to enable the communication between the frontend and the backend. The backend of BLPAD is executed within a Jetty server. The results computed by the different modules (i.e. display and deduction) are stored in Neo4J, which is a graph-oriented database. Our mathematical formulation is represented as a graph structure. Neo4J appeared to be the right choice to store our routing and scheduling results.

\section{CONCLUSIONS AND RESEARCH PERSPECTIVES}

This research work addresses a systemic analysis in order to identify the complexity of the operational coordination in an HHC ecosystem. Facing this complexity, we propose one prototype named BLPAD consisting of two modules: 1) to characterize the necessary information for coordination within an HHC system (DefPAD), and then 2) generate the optimal solution based on the modeled information (GenPAD).

In the future work, it will be interesting to develop a modeling language adapted to the definition of the HHC functioning. Then we wish to characterize a knowledge base from this capacity to be modeled in order to specify the coordination's needs. This formulation must be built in a flexible way to translate the various requirements of the actors. In addition to the techniques for exploiting the optimum in a deterministic situation, the research will have to make use of the uncertainty sources, or even the risks, acquired by using the model constructed during the first phase to study this solution in a non-deterministic situation.

\section{REFERENCES}

[1] V. Rajsiri, J.-P. Lorré, F. Bénaben, and H. Pingaud, "Knowledgebased system for collaborative process specification," Computers in Industry, vol. 61, no. 2, pp. 161-175, Feb. 2010.

[2] C. A. Hill and G. D. Scudder, "The use of electronic data interchange for supply chain coordination in the food industry," Journal of Operations Management, vol. 20, no. 4, pp. 375-387, Aug. 2002.

[3] K. M. McDonald et al., Closing the Quality Gap: A Critical Analysis of Quality Improvement Strategies (Vol. 7: Care Coordination). Agency for Healthcare Research and Quality (US), 2007.

[4] M. Gourgand, "La modélisation, la simulation et l'optimisation des flux dans les systèmes hospitaliers," Bulletin de la Société Française de Recherche Opérationnelle et d'Aide à la Décision, vol. 21, pp. 8 12,2008

[5] L. En-nahli, H. Allaoui, and I. Nouaouri, "A Multi-objective Modelling to Human Resource Assignment and Routing Problem for
Home Health Care Services," IFAC-PapersOnLine, vol. 48, no. 3, pp. 698-703, Jan. 2015.

[6] A. Castillo-Salazar, D. Landa-Silva, and R. Qu, "A survey of workforce scheduling and routing," presented at the Proceedings of the 9th International Conference on the Practice and Theory of Automated Timetabling (PATAT 2012), Oslo, Norway, 2012, pp. 283-302.

[7] E. Lanzarone and A. Matta, "Robust nurse-to-patient assignment in home care services to minimize overtimes under continuity of care," Operations Research for Health Care, vol. 3, no. 2, pp. 48-58, Jun. 2014

[8] A. Matta, S. Chahed, E. Sahin, and Y. Dallery, "Modelling home care organisations from an operations management perspective," Flex Serv Manuf J, vol. 26, no. 3, pp. 295-319, Sep. 2014.

[9] D. S. Mankowska, F. Meisel, and C. Bierwirth, "The home health care routing and scheduling problem with interdependent services," Health Care Manag Sci, vol. 17, no. 1, pp. 15-30, Mar. 2014.

[10] E. Lanzarone, A. Matta, and G. Scaccabarozzi, "A patient stochastic model to support human resource planning in home care," Production Planning \& Control, vol. 21, no. 1, pp. 3-25, Jan. 2010.

[11] G. Carello and E. Lanzarone, "A cardinality-constrained robust model for the assignment problem in Home Care services," European Journal of Operational Research, vol. 236, no. 2, pp. 748762, Jul. 2014.

[12] Z. Yuan and A. Fügenschuh, Home health care scheduling: a case study. Helmut-Schmidt-Univ., Professur für Angewandte Mathematik, 2015.

[13] G. Hiermann, M. Prandtstetter, A. Rendl, J. Puchinger, and G. R. Raidl, "Metaheuristics for solving a multimodal home-healthcare scheduling problem," Cent Eur J Oper Res, vol. 23, no. 1, pp. 89113, Mar. 2015

[14] P. A. Maya Duque, M. Castro, K. Sörensen, and P. Goos, "Home care service planning. The case of Landelijke Thuiszorg," European Journal of Operational Research, vol. 243, no. 1, pp. 292-301, May 2015.

[15] G. Du, X. Liang, and C. Sun, "Scheduling Optimization of Home Health Care Service Considering Patients' Priorities and Time Windows," Sustainability, vol. 9, no. 2, p. 253, Feb. 2017.

[16] A. Trautsamwieser and P. Hirsch, "A Branch-Price-and-Cut approach for solving the medium-term home health care planning problem," Networks, vol. 64, no. 3, pp. 143-159, Oct. 2014.

[17] K.-D. Rest and P. Hirsch, "Daily scheduling of home health care services using time-dependent public transport," Flex Serv Manuf J, vol. 28, no. 3, pp. 495-525, Sep. 2016.

[18] Y. Shao, J. F. Bard, and A. I. Jarrah, "The therapist routing and scheduling problem," IIE Transactions, vol. 44, no. 10, pp. 868-893, Oct. 2012.

[19] R. Redjem and E. Marcon, "Operations management in the home care services: a heuristic for the caregivers' routing problem," Flex Serv Manuf J, vol. 28, no. 1-2, pp. 280-303, Jun. 2016.

[20] M. Gendreau, A. Hertz, G. Laporte, and M. Stan, "A Generalized Insertion Heuristic for the Traveling Salesman Problem with Time Windows," Operations Research, vol. 46, no. 3, pp. 330-335, Jun. 1998

[21] D. Bertsimas and M. Sim, "The Price of Robustness," Operations Research, vol. 52, no. 1, pp. 35-53, Feb. 2004.

[22] B. Yuan, R. Liu, and Z. Jiang, "A branch-and-price algorithm for the home health care scheduling and routing problem with stochastic service times and skill requirements," International Journal of Production Research, vol. 53, no. 24, pp. 7450-7464, Dec. 2015.

[23] M. Issabakhsh, S.-M. Hosseini-Motlagh, M.-S. Pishvaee, and M. Saghafi Nia, "A Vehicle Routing Problem for Modeling Home Healthcare: a Case Study," International Journal of Transportation Engineering, vol. 5, no. 3, pp. 211-228, Jan. 2018.

[24] C. Rodriguez, T. Garaix, X. Xie, and V. Augusto, "Staff dimensioning in homecare services with uncertain demands," International Journal of Production Research, vol. 53, no. 24, pp. 7396-7410, Dec. 2015

[25] S. Yalçındağ, A. Matta, E. Şahin, and J. G. Shanthikumar, "The patient assignment problem in home health care: using a data-driven method to estimate the travel times of care givers," Flex Serv Manuf $J$, vol. 28, no. 1-2, pp. 304-335, Jun. 2016.

[26] M. P. Wand and M. C. Jones, Kernel Smoothing. CRC Press, 1994.

[27] L. Zhang et al., "Home Health Care Daily Planning Considering the Satisfaction of all the Stakeholders."., in press 